Pacific

Journal of

Mathematics

A SPHERE THEOREM FOR 2-DIMENSIONAL CAT(1)-SPACES

KOICHI NAGANO 


\title{
A SPHERE THEOREM FOR 2-DIMENSIONAL CAT(1)-SPACES
}

\author{
KOICHI NAGANO
}

\begin{abstract}
We study sphere theorems for compact, geodesically complete 2-dimensional CAT(1)-spaces. As one of the main results, for compact, geodesically complete, 2-dimensional CAT(1)-spaces, we obtain the optimal volume condition to ensure being homeomorphic to the 2-sphere.
\end{abstract}

\section{Introduction.}

The problems of sphere theorems in Riemannian geometry have yielded the beautiful results and the fruitful techniques for the study of global geometry (cf. [22]). The main purpose of this paper is to study sphere theorems for CAT(1)-spaces: When are CAT(1)-spaces homeomorphic to the sphere?

The notion of $\operatorname{CAT}(\kappa)$-spaces is introduced by Gromov $([\mathbf{1 1}])$ based on Alexandrov's original notion, i.e., spaces with curvature bounded above by $\kappa \in \mathbb{R}$. The research for CAT(1)-spaces is important since the space of directions at a given point in a $\operatorname{CAT}(\kappa)$-space, which has the most local geometric information, is a CAT(1)-space. Furthermore, the ideal boundary of a given CAT(0)-space (the so-called, Hadamard space), which has the most global one, is a CAT(1)-space. In addition, all spherical buildings are CAT(1)-spaces (cf. [13], [23]).

Throughout this paper, we always assume that $\mathrm{CAT}(\kappa)$-spaces have the local compactness and the geodesical completeness. Nevertheless, the local metric structure may be complicated. For example, it is known by Kleiner that a $\operatorname{CAT}(\kappa)$-space $X$ may admit no triangulation even if $X$ is 2-dimensional (cf. [12], [14]). We require the careful treatment of the local structure.

If $X$ is a compact, geodesically complete CAT(1)-space, then the diameter of $X$ is not smaller than $\pi$. There exist many examples of compact, geodesically complete CAT(1)-spaces possessing the minimal diameter $\pi$ which are not homeomorphic to each other: Ballmann and Brin [5] have classified the isometry classes of the 2-dimensional spherical polyhedra in some sense which are such CAT(1)-spaces of the minimal diameter $\pi$.

In this paper, we shall study volume sphere theorems for compact, geodesically complete CAT(1)-spaces. 
1.1. CAT $(\kappa)$-spaces. We first state the precise definition of CAT $(\kappa)$-spaces in this paper. We refer to [1], [2], [3], and [7] for the fundamental properties of $\mathrm{CAT}(\kappa)$-spaces, more generally, of spaces with curvature bounded above.

For $\kappa \in \mathbb{R}$, we set $D_{\kappa}:=\operatorname{diam} M_{\kappa}^{n}$, i.e., the diameter of the $n$-dimensional, complete, simply connected model space $M_{\kappa}^{n}$ with constant sectional curvature $\kappa$.

Let $\left(X, d_{X}\right)$ be a complete metric space. We say that $X$ is a $\mathrm{CAT}(\kappa)$-space if $X$ satisfies the following:

(i) $\left(D_{\kappa}\right.$-geodesic) Every two points $x, y \in X$ with $d_{X}(x, y)<D_{\kappa}$ are joined by a minimizing geodesic $x y$.

(ii) (CAT $(\kappa)$-property) For an arbitrary geodesic triangle $\triangle \subset X$ with perimeter $<2 D_{\kappa}$, we have the comparison triangle $\widetilde{\triangle} \subset M_{\kappa}^{2}$ (with the same side lengths as $\triangle$ ) such that $d_{X}(x, y) \leq d_{M_{\kappa}^{2}}(\widetilde{x}, \widetilde{y})$ for every pair $x, y \in \triangle$ and the corresponding points $\widetilde{x}, \widetilde{y} \in \widetilde{\triangle}$.

We now note the following important properties of CAT $(\kappa)$-spaces:

(i) The convexity radii of all points are not smaller than $D_{\kappa} / 2$.

(ii) The injectivity radii of all points are not smaller than $D_{\kappa}$. In particular, the $D_{\kappa}$-neighborhood of a given point is contractible.

The first one is also related to the property that $d_{X}$ is (semi) convex.

1.2. Simple examples of CAT(1)-spaces. Next, we provide simple examples of CAT(1)-spaces. We remark that, if $X$ is a CAT $(\kappa)$-space for some $\kappa>0$, then $\sqrt{\kappa} X:=\left(X, \sqrt{\kappa} d_{X}\right)$ is a $\operatorname{CAT}(1)$-space.

We here recall Reshetnyak's gluing lemma ([19], cf. [7]): The space constructed by gluing CAT $(\kappa)$-spaces isometrically along proper convex subsets is again a $\operatorname{CAT}(\kappa)$-space.

Example 1.1. Here, all $X$ in (i)-(v) are compact, geodesically complete CAT(1)-spaces:

(i) Let $X$ be the $n$-dimensional sphere $\mathbb{S}^{n}(r)$ with radius $r>0$. Then, for any $r \geq 1$, the space $X=\mathbb{S}^{n}(r)$ is a $\mathrm{CAT}(1)$-space.

(ii) We take mutually antipodal points $p, \hat{p} \in \mathbb{S}^{n}(1)$ and the closed interval $[0, \pi]$. Let $X$ be the quotient space obtained by gluing $\mathbb{S}^{n}(1)$ and $[0, \pi]$ along $p=\{0\}$ and $\hat{p}=\{\pi\}$. Then, $X$ is a CAT(1)-space. (cf. Figure 1.)

(iii) We prepare $\mathbb{S}^{n}(1)$ and the (distinct) closed unit $n$-hemisphere $\mathbb{H} \mathbb{S}^{n}(1)$. Let $X$ be the quotient space obtained by gluing $\mathbb{S}^{n}(1)$ and $\mathbb{H} \mathbb{S}^{n}(1)$ along their equators. Then, $X:=\mathbb{S}^{n}(1) \sqcup \mathbb{H}^{n}(1) /$ equator is a CAT(1)-space. (cf. Figure 2.)

(iv) Let $X$ be the $n$-dimensional real projective space $\mathbb{R P}^{n}(r)$ as the quotient for $\mathbb{S}^{n}(r)$ by the standard $\mathbb{Z}_{2}$-action. Then, for any $r \geq 2$, the space $X=\mathbb{R P}^{n}(r)$ is a $\mathrm{CAT}(1)$-space. 
(v) Let $X=\mathbb{T}^{2}(2 \pi \times 2 \pi)=\mathbb{S}^{1}(1) \times \mathbb{S}^{1}(1)$ be the flat torus whose universal covering space has the fundamental domain of the flat $(2 \pi \times 2 \pi)$-square. Then, $X$ is a $\mathrm{CAT}(1)$-space.

More generally, complete, smooth Riemannian manifolds with sectional curvature uniformly bounded above by 1 and of injectivity radii bounded below by $D_{1}$ are CAT(1)-spaces.

1.3. Main theorems. Let $X$ be a locally compact, geodesically complete $\operatorname{CAT}(\kappa)$-space. For $n \in \mathbb{N}$, we denote by $\bar{X}^{n} \subset X$ the set of all points whose open $t$-balls have the Hausdorff dimension $n$ for any sufficiently small $t>0$.

Throughout this paper, dim denotes the Hausdorff dimension, and $\mathcal{H}^{n}(\cdot)$ the $n$-dimensional Hausdorff measure. In addition, the symbol $\vartheta_{\alpha, \beta, \ldots}(\epsilon)$ denotes the positive function depending only on $\alpha, \beta, \ldots$ with $\lim _{\epsilon \rightarrow 0} \vartheta_{\alpha, \beta, \ldots}(\epsilon)$ $=0$.

In [15], from the CAT(1)-property, the author shows the following: For given $n \in \mathbb{N}$, let $X$ be a compact, geodesically complete CAT(1)-space satisfying $X=\bar{X}^{n}$. Then, $\mathcal{H}^{n}(X) \geq \mathcal{H}^{n}\left(\mathbb{S}^{n}(1)\right)$. Moreover, the equality holds if and only if $X$ is isometric to $\mathbb{S}^{n}(1)$.

Furthermore, the author proves the following sphere theorem $([\mathbf{1 5}])$ : For given $n \in \mathbb{N}$, we have a positive number $\bar{\epsilon}_{n}>0$ satisfying the following: We assume that $X$ is a compact, geodesically complete CAT(1)-space such that:

(i) $X=\bar{X}^{n}$.

(ii) The following holds for $\epsilon \in\left(0, \bar{\epsilon}_{n}\right)$ :

$$
\mathcal{H}^{n}(X)<\mathcal{H}^{n}\left(\mathbb{S}^{n}(1)\right)+\epsilon .
$$

Then, there exists a bi-Lipschitz homeomorphism between $X$ and $\mathbb{S}^{n}(1)$ such that the Lipschitz constants are contained in $\left(1-\vartheta_{n}(\epsilon), 1+\vartheta_{n}(\epsilon)\right)$.

We remark that the above Assumption (i) is essential because of Example 1.1.(ii).

We now concentrate on the case $n=2$. We consider how much the above volume condition (1.1) can be relaxed.

As one of the main results, we prove the following sphere theorem for 2-dimensional CAT(1)-spaces:

Theorem A. Let $X$ be a compact, geodesically complete CAT(1)-space satisfying $X=\bar{X}^{2}$ and

$$
\mathcal{H}^{2}(X)<(3 / 2) \mathcal{H}^{2}\left(\mathbb{S}^{2}(1)\right)
$$

Then, $X$ is homeomorphic to a 2-dimensional sphere $\mathbb{S}^{2}$.

Remark 1.2. The condition (1.2) is optimal for Theorem A because, for $X=\mathbb{S}^{2}(1) \sqcup \mathbb{H} \mathbb{S}^{2}(1) /$ equator as in Example 1.1.(iii), we see that $X=\bar{X}^{2}$, $\mathcal{H}^{2}(X)=(3 / 2) \mathcal{H}^{2}\left(\mathbb{S}^{2}(1)\right)$, and that $X$ is not homeomorphic to $\mathbb{S}^{2}$. 
Remark 1.3. Without the assumption $X=\bar{X}^{2}$, we can observe an embedding of $\mathbb{S}^{2}$ into a CAT(1)-space of the Hausdorff dimension $\leq 2$. In Section 5 , we shall prove the following: Let $X$ be a locally compact, geodesically complete CAT(1)-space of the Hausdorff dimension $\leq 2$ with $\bar{X}^{2} \neq \emptyset$ such that $\mathcal{H}^{2}(X)<(3 / 2) \mathcal{H}^{2}\left(\mathbb{S}^{2}(1)\right)$. Then, there exists a locally convex subset $Y \subset X$ such that $Y$ is a 2-dimensional Lipschitz manifold homeomorphic to $\mathbb{S}^{2}$. Actually, $Y=\bar{X}^{2}$, and $Y$ is a compact, geodesically complete CAT(1)-space with respect to the interior distance in $Y$.

Remark 1.4. At the same time proving Theorem A, we observe the following for smooth Riemannian manifolds: Let $M$ be a compact, smooth Riemannian manifold of dimension $n$ which is also a CAT(1)-space. Assume that $\mathcal{H}^{n}(M)<(3 / 2) \mathcal{H}^{n}\left(\mathbb{S}^{n}(1)\right)$. Then, $M$ is homeomorphic to an $n$-dimensional sphere $\mathbb{S}^{n}$.

In smooth Riemannian case, Coghlan and Itokawa [9] have obtained the result related to Theorem A as follows: Let $M$ be a compact, simply connected Riemannian manifold of even dimension $m$. Assume that $M$ has positive sectional curvature with uniformly bounded above by $\kappa$, and that its volume $\operatorname{vol}(M)$ satisfies $\operatorname{vol}(M) \leq(3 / 2) \operatorname{vol}\left(\mathbb{S}^{m}(1)\right) / \kappa^{m / 2}$. Then, $M$ is homeomorphic to $\mathbb{S}^{m}(1)$.

In our general case, we furthermore obtain the following:

Theorem B. Let $X$ be a compact, geodesically complete CAT(1)-space satisfying $X=\bar{X}^{2}$ and $\mathcal{H}^{2}(X)=(3 / 2) \mathcal{H}^{2}\left(\mathbb{S}^{2}(1)\right)$. Then, $X$ is either homeomorphic to $\mathbb{S}^{2}$ or isometric to $\mathbb{S}^{2}(1) \sqcup \mathbb{H} \mathbb{S}^{2}(1) /$ equator

In Section 5, we also investigate the number of the homotopy types of CAT(1)-spaces: For $n \in \mathbb{N}$ and $V>0$, let us denote by $\mathcal{C}(n, V)$ the isometry classes of all compact, geodesically complete CAT(1)-spaces such that $X=$ $\bar{X}^{n}$ and $\mathcal{H}^{n}(X) \leq V$. Then, the number of the homotopy types of $\mathcal{C}(n, V)$ is bounded above by a constant depending only on $n$ and $V$.

1.4. The outline of our proofs of main theorems. First, we simply review the convergence theorem, which is studied in [15], for compact, geodesically complete CAT $(\kappa)$-spaces: For a given CAT $(\kappa)$-space with weak singularities in some sense, let us consider the other CAT $(\kappa)$-space sufficiently close to it with respect to the Gromov-Hausdorff distance. Then, we have an almost isometry, and hence a bi-Lipschitz homeomorphism between them. (See Section 2.)

We also have volume comparison for CAT $(\kappa)$-spaces (cf. [15]), i.e., the opposite inequalities to the well-known of Bishop type and of Bishop-Gromov type for smooth Riemannian manifolds with curvature bounded below.

Let $X$ be the 2-dimensional one as in Theorem A. Then, using the volume comparison, (1.2), and the convergence theorem $([\mathbf{1 5}])$, we can prove 
the following: Every point in $X$ as in Theorem A has a neighborhood homeomorphic to a 2-dimensional open disk, in particular, $X$ is a 2-dimensional topological manifold. More generally, for a given point, we also obtain the optimal local volume growth condition to possess a neighborhood homeomorphic to a 2-disk in Section 3. Namely, we obtain the following:

Proposition C. For $\kappa \in \mathbb{R}$, let us denote by $X$ a locally compact, geodesically complete $\mathrm{CAT}(\kappa)$-space. Assume that a point $x \in \bar{X}^{2}$ satisfies the following: $\mathcal{H}^{2}\left(B_{x}(T ; X)\right) / \omega_{\kappa}^{2}(T)<3 / 2$ for some $T \in\left(0, D_{\kappa}\right]$. Then, there exists a positive number $t=t_{x}>0$ such that $B_{x}(t ; X)$ is homeomorphic to a 2-dimensional, Euclidean open disk $B^{2} \subset \mathbb{R}^{2}$.

Here, we denote by $B_{x}(t ; X)$ the open $t$-ball centered at $x \in X$, and by $\omega_{\kappa}^{2}(T)$ the 2-dimensional Hausdorff measure of a $T$-ball in $M_{\kappa}^{2}$.

Remark 1.5. The local structure of locally compact, geodesically complete $\mathrm{CAT}(\kappa)$-spaces, especially of dimension 2, has been already studied by Kleiner, Burago and Buyalo [8]. Proposition $\mathrm{C}$ can be also proved by using their studies mentioned in Section 3 in [8].

Furthermore, (1.2) implies that $X$ as in Theorem A can be covered by two contractible open balls. Then, the Jordan curve theorem concludes that $X$ is homeomorphic to a 2 -sphere. Thereby, we prove Theorem A.

We next consider $X$ as in Theorem B. We denote by $\left\{z_{i}\right\} \subset X$ a maximal $\pi$-discrete set, i.e., $d_{X}\left(z_{i}, z_{j}\right) \geq \pi$ for $i \neq j$. Then, the volume comparison and the assumption $\mathcal{H}^{2}(X)=(3 / 2) \mathcal{H}^{2}\left(\mathbb{S}^{2}(1)\right)$ in Theorem B imply that $\sharp\left\{z_{i}\right\}=2$ or 3 for any maximal $\pi$-discrete set $\left\{z_{i}\right\} \subset X$. If $\sharp\left\{z_{i}\right\}=2$ for any such $\left\{z_{i}\right\} \subset X$, then $X$ is homeomorphic to a 2 -sphere from the similar idea to that in the proof of Theorem A. Assume that $\sharp\left\{z_{i}\right\}=3$ for some maximal $\pi$-discrete set $\left\{z_{i}\right\} \subset X$. Then, from a volume rigidity, $X$ is the union of the closed convex subsets isometric to the unit hemisphere with pole $z_{i}, i=1,2,3$. Considering how the boundaries of the unit hemispheres meet each other, we can show that $X$ is either homeomorphic to a 2 -sphere or isometric to $\mathbb{S}^{2}(1) \sqcup \mathbb{H} \mathbb{S}^{2}(1)$ / equator. In this way, we prove Theorem B.

1.5. The organization of this paper. The organization of this paper is as follows:

Section 2: We discuss the fundamental properties and the known facts for $\mathrm{CAT}(\kappa)$-spaces.

Section 3: We observe the existence of 2-disk neighborhoods in $\operatorname{CAT}(\kappa)$ spaces, and show Proposition C.

Section 4: We prove Theorems A and B.

Section 5: We research some topological embeddings into CAT $(\kappa)$-spaces of the Hausdorff dimension $\leq 2$. 
Section 6: We provide some prospects for the study of CAT $(\kappa)$-spaces from a topological viewpoint.

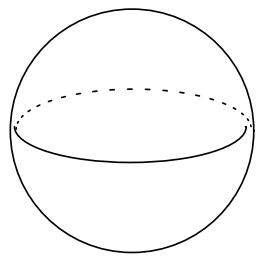

$\mathbb{S}^{n}(1)$

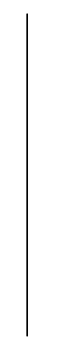

$[0, \pi]$

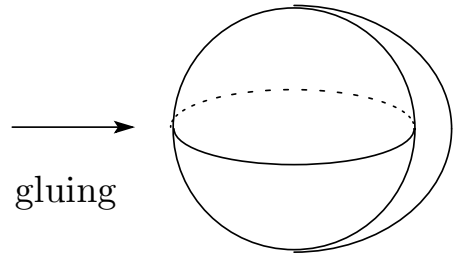

$X$

Figure 1. Example 1.1.(ii).

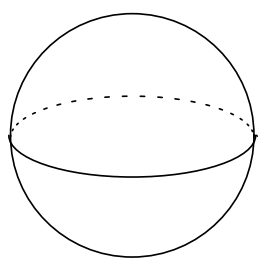

$\mathbb{S}^{n}(1)$

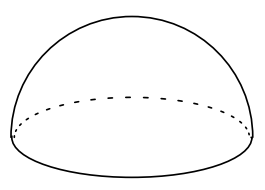

$\mathbb{H} \mathbb{S}^{n}(1)$

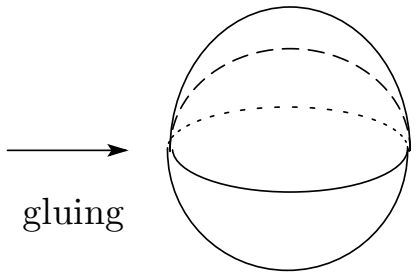

$X$

Figure 2. Example 1.1.(iii).

\section{Preliminaries.}

In this section, we list the basic properties and the known facts of CAT $(\kappa)$ spaces, spaces with curvature bounded above, which will be needed in the subsequent sections.

Let $\left(X, d_{X}\right)$ be a complete metric space. We denote by $B_{x}(t ; X)\left(\operatorname{resp} . \bar{B}_{x}(t ; X)\right)$ the open (resp. closed) metric ball with radius $t>0$ centered at $x \in X$.

2.1. Spaces with curvature bounded above and various radii. For $\kappa \in \mathbb{R}$, we say that $X$ is an Alexandrov space with curvature bounded above by $\kappa$ if $X$ is locally $\operatorname{CAT}(\kappa)$, i.e., if for every $x \in X$ there exists a positive number $R=R_{x} \in\left(0, D_{\kappa} / 2\right]$ such that $\bar{B}_{x}(R ; X)$ is a $\operatorname{CAT}(\kappa)$-space. Then, we remark that, $\bar{B}_{x}(R ; X)$ is a convex subset in $X$ for $R \in\left(0, D_{\kappa} / 2\right]$.

Let $x \in X$ be a point in an Alexandrov spaces with curvature $\leq \kappa$. We then define various radii at $x$ as follows: 
- The injectivity radius at $x, \operatorname{Inj} \operatorname{Rad}(x)$, is defined as the supremum of $R>0$ satisfying the following: For every $y \in B_{x}(R ; X), x$ and $y$ are joined by the unique minimizing geodesic $x y$.

- The $C A T(\kappa)$-radius at $x, \mathrm{CAT}_{\kappa} \operatorname{Rad}(x)$, the supremum of $R \in\left(0, D_{\kappa} / 2\right]$ satisfying: $\bar{B}_{x}(R ; X)$ is a $\mathrm{CAT}(\kappa)$-space.

- The comparable radius at $x, \operatorname{Comp}_{\kappa} \operatorname{Rad}(x)$, the supremum of $R \in$ $\left(0, D_{\kappa}\right]$ satisfying: For every two points $y, z \in B_{x}(R ; X)$ which satisfy $d_{X}(x, y)+d_{X}(y, z)+d_{X}(z, x)<2 D_{\kappa}$, there exists a geodesic triangle $\triangle(x, y, z) \subset X$ with the vertices $x, y, z$ such that $\triangle(x, y, z)$ has the $\mathrm{CAT}(\kappa)$-property.

Then, by definition, we have

$$
0<\operatorname{CAT}_{\kappa} \operatorname{Rad}(x) \leq \operatorname{Comp}_{\kappa} \operatorname{Rad}(x) \leq \operatorname{Inj} \operatorname{Rad}(x) .
$$

Moreover, if $X$ itself is a $\mathrm{CAT}(\kappa)$-space, then for any $x \in X$ we have

$$
2 \mathrm{CAT}_{\kappa} \operatorname{Rad}(x)=\operatorname{Comp}_{\kappa} \operatorname{Rad}(x)=D_{\kappa} .
$$

2.2. Spaces of directions and the tangent cones. For complete metric space $X$, we say that $X$ is geodesically complete if every (nontrivial) geodesic is contained in a geodesic whose domain of the parameterization is a whole real line.

For a while, let $X$ denote a locally compact, geodesically complete Alexandrov space with curvature $\leq \kappa$.

For $x \in X$, we write $\Sigma_{x} X:=\{x y \mid y \in X \backslash\{x\}\} /{ }_{\angle_{x}=0}$, called the space of directions at $x$, where $\angle_{x}$ is the angle at $x$. The direction $v_{x y} \in \Sigma_{x} X$ often denotes $[x y] \in \Sigma_{x} X$. We write $C_{x} X$, called the tangent cone at $x$, as the Euclidean cone $\Sigma_{x} X \times[0, \infty) / \Sigma_{x} X \times\{0\}$. Note that $\Sigma_{x} X$ is a compact, geodesically complete CAT(1)-space, and that $C_{x} X$ is a locally compact, geodesically complete $\mathrm{CAT}(0)$-space $([\mathbf{1}],[\mathbf{2}])$. We also remark that $\left(C_{x} X, \star\right)$ is isometric to the (pointed) Gromov-Hausdorff limit of $\left(\frac{1}{t} X, x\right)$ as $t \searrow 0$, where $\star \in C_{x} X$ is the vertex of the cone.

2.3. Branch points and their measure. We here introduce the notion of branch points by Otsu and Tanoue ([17]) for representing singularities in spaces with curvature bounded above.

For $\delta>0$ and $x \in X$, a point $z \in X$ is a $\delta$-branch point of $x$ if the following holds: $\operatorname{diam}\left\{v \in \Sigma_{z} X \mid \angle_{z}\left(v_{z x}, v\right)=\pi\right\} \geq \delta$. We denote by $S_{x, \delta}$ the set of all $\delta$-branch points of $x$. Furthermore, we define $S_{\delta}(X):=\cup\left\{S_{x, \delta} \mid x \in X\right\}$, called $\delta$-branch points in $X$. Note that both $S_{x, \delta}$ and $S_{\delta}(X)$ are closed in $X$ for any $x \in X$ and $\delta>0([\mathbf{1 7}],[\mathbf{1 5}])$.

For given positive integer $n \in \mathbb{N}$, we write

$$
\begin{gathered}
X^{n}:=\left\{x \in X \mid \operatorname{dim} \Sigma_{x} X=n-1\right\}, \\
\bar{X}^{n}:=\left\{x \in X \mid \operatorname{dim} B_{x}(t ; X)=n \text { for any sufficiently small } t>0\right\},
\end{gathered}
$$




$$
\widehat{X}^{n}:=\left\{x \in X \mid \operatorname{dim} B_{x}(t ; X) \leq n \text { for some } t>0\right\},
$$

where $\bar{X}^{n}$ is the same one as that defined in Section 1. Furthermore, as some singular sets, we write $S_{X}^{n}:=\left\{x \in \widehat{X}^{n} \mid \Sigma_{x} X \neq \mathbb{S}^{n-1}(1)\right\}$.

Otsu and Tanoue ([16], [17]) study the Hausdorff measures of singular points as follows:

Theorem $2.1([\mathbf{1 6}],[\mathbf{1 7}])$. For a given positive integer $n \in \mathbb{N}$, we assume that $B_{x}(T ; X) \subset \widehat{X}^{n}$ for some $T \in\left(0, \mathrm{CAT}_{\kappa} \operatorname{Rad}(x)\right)$. Then, we obtain the following:

(i) $\mathcal{H}^{n}\left(S_{x, \delta} \cap B_{x}(T ; X)\right)=0$ for any $\delta>0$.

(ii) $\mathcal{H}^{n}\left(S_{X}^{n} \cap B_{x}(T ; X)\right)=0$.

In particular, if $\mathcal{H}^{n}\left(B_{x}(t ; X)\right)>0$ also holds for $t \in(0, T)$, then there exists a point $y \in B_{x}(t ; X)$ in an $\mathcal{H}^{n}$-full measure subset in $B_{x}(t ; X)$ such that $\Sigma_{y} X=\mathbb{S}^{n-1}(1)$.

Here, we remark the following $([\mathbf{1 5}]): \bar{X}^{n} \subset X^{n}$ holds for given $n \in \mathbb{N}$. Moreover, if $X=\widehat{X}^{n}$ also holds, then $\bar{X}^{n}=X^{n}$.

Furthermore, the author $([\mathbf{1 5}])$ verifies the following:

Lemma $2.2([\mathbf{1 5}])$. For given $n \in \mathbb{N}$, assume that $B_{x}(T ; X) \subset \bar{X}^{n}$ for some $x \in X$ and $T>0$. Then, we obtain $\Sigma_{x} X={\overline{\left(\Sigma_{x} X\right)}}^{n-1}$ and $C_{x} X={\overline{\left(C_{x} X\right)}}^{n}$.

2.4. Convention. For metric spaces $Y$ and $Z$, a map $f_{1}: Y \rightarrow Z$ is called an expanding map if $d_{Z}\left(f_{1}\left(y_{1}\right), f_{1}\left(y_{2}\right)\right) \geq d_{Y}\left(y_{1}, y_{2}\right)$ holds for every $y_{1}, y_{2} \in$ $Y$.

For $\vartheta>0$, a surjective map $f_{2}: Y \rightarrow Z$ is said to be a $\vartheta$-almost isometry if $\left|d_{Z}\left(f_{2}\left(y_{1}\right), f_{2}\left(y_{2}\right)\right)-d_{Y}\left(y_{1}, y_{2}\right)\right|<\vartheta d_{Y}\left(y_{1}, y_{2}\right)$ for every $y_{1}, y_{2} \in Y$. We note that: If $\vartheta<1$, then the map $f_{2}$ is a bi-Lipschitz homeomorphism. Furthermore, if $f_{2}$ is a $\vartheta$-almost isometry for any $\vartheta>0$, then $f_{2}$ is an isometry.

2.5. Convergence theorems. We now denote by $d_{G H}$ the Gromov-Hausdorff distance (cf. [10]).

The following is the convergence theorem which is mentioned in Section 1 for spaces with only weak singularities:

Theorem 2.3 ([15]). For given constants $\kappa \in \mathbb{R}, n \in \mathbb{N}$, and $R_{0}>0$, we find a positive constant $\bar{\delta}=\bar{\delta}_{n}>0$ with the following properties: Let $X$ denote a compact, geodesically complete Alexandrov space with curvature $\leq \kappa$ satisfying $X=\bar{X}^{n}$ and $S_{\delta}(X)=\emptyset$ for $\delta \in(0, \bar{\delta})$. We then find an $\bar{\epsilon}=\bar{\epsilon}_{\kappa, n, R_{0}, \delta, X}>0$ satisfying the following: If $Y$ is a compact, geodesically complete Alexandrov space with curvature $\leq \kappa$ such that $\operatorname{CAT}_{\kappa} \operatorname{Rad}(y) \geq R_{0}$ for any $y \in Y$, and that $d_{G H}(X, Y)<\epsilon$ for $\epsilon \in(0, \bar{\epsilon})$, then there exists a $\left(\vartheta_{n}(\delta)+\vartheta_{\kappa, n, R_{0}, X}(\epsilon)\right)$-almost isometry $\Psi: Y \rightarrow X$. 
Remark 2.4. The construction of the almost isometry discussed in [15] guarantees that there also exists an almost isometry between some $d_{G H^{-}}$ close local parts with only weak singularities.

In [15], using Theorem 2.3, the author studies volume convergence theorems for Alexandrov spaces with curvature bounded above. As one of them, we obtain the following local volume regularity:

Theorem 2.5 ([15]). Let $X$ be a locally compact, geodesically complete Alexandrov space with curvature $\leq \kappa$. If $x \in \bar{X}^{n}$ holds for given $n \in \mathbb{N}$, then we have

$$
\lim _{t \rightarrow 0} \frac{\mathcal{H}^{n}\left(B_{x}(t ; X)\right)}{t^{n}}=\mathcal{H}^{n}\left(B_{\star}\left(1 ; C_{x} X\right)\right) \in(0, \infty) .
$$

Here, $\star \in C_{x} X$ is the vertex of the Euclidean cone.

\subsection{Volume comparison for spaces with curvature bounded above.}

For $\kappa \in \mathbb{R}$ and $n \in \mathbb{N}$, we denote by $\omega_{\kappa}^{n}(t)$ the $n$-dimensional Hausdorff measure of a $t$-ball in $M_{\kappa}^{n}$. Let $X$ be a locally compact, geodesically complete Alexandrov space with curvature $\leq \kappa$.

The following absolute volume comparison can be obtained by the CAT $(\kappa)$ property $([\mathbf{1 5}])$ :

Proposition $2.6([\mathbf{1 5}])$. For given $n \in \mathbb{N}$, we have

$$
\mathcal{H}^{n}\left(B_{x}(t ; X)\right) \geq \omega_{\kappa}^{n}(t)
$$

for any $x \in \bar{X}^{n}$ and $t \in\left[0, \operatorname{Comp}_{\kappa} \operatorname{Rad}(x)\right]$.

Furthermore, assume that $B_{x}(t ; X) \subset \bar{X}^{n}$ for $t \in\left[0, \operatorname{CAT}_{\kappa} \operatorname{Rad}(x)\right]$. Then, the equality in (2.1) holds if and only if the convex set $B_{x}(t ; X)$ is isometric to $B_{\bar{x}}\left(t ; M_{\kappa}^{n}\right)$ for a given point $\bar{x} \in M_{\kappa}^{n}$.

In fact, the inequality (2.1) is obtained by the following:

Lemma $2.7([\mathbf{1 5}])$. For given $n \in \mathbb{N}$, we take a point $x \in \bar{X}^{n}$. Then, there exists an expanding map $g_{x}: \mathbb{S}^{n-1}(1) \rightarrow \Sigma_{x} X$.

We now define $\partial B_{x}(t ; X):=\left\{y \in X \mid d_{X}(x, y)=t\right\}$. We then provide the coarea formula for the distance functions (cf. [15]):

Lemma 2.8. For given $n \in \mathbb{N}$, assume that $\mathcal{H}^{n}\left(B_{x}(T ; X)\right)<\infty$ for $x \in X$ and $T \in\left(0, \operatorname{Comp}_{\kappa} \operatorname{Rad}(x)\right)$. Then, we have

$$
\mathcal{H}^{n}\left(B_{x}(T ; X)\right)=\int_{0}^{T} \mathcal{H}^{n-1}\left(\partial B_{x}(t ; X)\right) d t .
$$

The following relative volume comparison can be also obtained by Lemma 2.8 and the $\operatorname{CAT}(\kappa)$-property $([\mathbf{1 5}])$ : 
Proposition 2.9 ([15]). For given $n \in \mathbb{N}$ and $x \in \bar{X}^{n}$, let us define the function $F:\left(0, \operatorname{Comp}_{\kappa} \operatorname{Rad}(x)\right] \rightarrow[1,+\infty]$ as

$$
F(t):=\mathcal{H}^{n}\left(B_{x}(t ; X)\right) / \omega_{\kappa}^{n}(t) .
$$

Then, $F$ is monotone non-decreasing as $t \nearrow$.

Remark 2.10. In general, $F(t)$ as in Proposition 2.9 does not necessarily converge to 1 as $t \searrow 0$. More precisely, by Lemma 2.8 and Theorem 2.5, we obtain the following (cf. [15]):

$$
F(t)=\frac{\mathcal{H}^{n}\left(B_{x}(t ; X)\right)}{t^{n}} \frac{t^{n}}{\omega_{\kappa}^{n}(t)} \rightarrow \frac{\mathcal{H}^{n}\left(B_{\star}\left(1 ; C_{x} X\right)\right)}{\omega_{0}^{n}(1)}=\frac{\mathcal{H}^{n-1}\left(\Sigma_{x} X\right)}{\mathcal{H}^{n-1}\left(\mathbb{S}^{n-1}(1)\right)}
$$

as $t \searrow 0$, where $\star$ is the vertex of $C_{x} X$.

\section{Two dimensional disk neighborhoods in spaces with curvature bounded above.}

In this section, we observe some topological properties of spaces with curvature bounded above. We also prove Proposition C.

For $\kappa \in \mathbb{R}$, let $X$ denote a locally compact, geodesically complete Alexandrov space with curvature $\leq \kappa$, and let $x \in X$ satisfy $x \in \bar{X}^{2}$. We now consider its space of directions $\Sigma_{x} X$. Then, $\operatorname{dim} \Sigma_{x} X=1$, and hence $\Sigma_{x} X$ has a structure of finite graph equipped with the vertex set containing $S_{\pi}\left(\Sigma_{x} X\right)$ (cf. Lemma 2.9 in [14]). If $\Sigma_{x} X$ is homeomorphic to $\mathbb{S}^{1}$ and its length is sufficiently close to $2 \pi$, then we see that $x \in X$ has a 2 -dimensional disk neighborhood. This follows from Theorem 3.1 in [8], which is obtained by Kleiner, stated by Burago and Buyalo.

More generally, we obtain the following:

Proposition 3.1. Let $x \in X$ be a point in a locally compact, geodesically complete Alexandrov space $X$ with curvature $\leq \kappa$ such that $\Sigma_{x} X$ is homeomorphic to $\mathbb{S}^{1}$. Then, we have a positive number $t=t_{x}>0$ such that $B_{x}(t ; X)$ is bi-Lipschitz homeomorphic to $B_{\star}\left(t ; C_{x} X\right)$; in particular, $B_{x}(t ; X)$ is homeomorphic to a 2-dimensional open disk $B^{2} \subset \mathbb{R}^{2}$.

Remark 3.2. Let $x \in X$ be as in Proposition 3.1. Then, as a consequence, we see that $x \in \bar{X}^{2}$.

Remark 3.3. Proposition 3.1 can be proved by using Theorem 3.1 in [8] since $C_{x} X$ is the Euclidean cone over a circle. The details are omitted.

Remark 3.4. Now, let us consider an Alexandrov space $X$ with curvature $\leq \kappa$ so that $X$ is a 2-dimensional topological manifold without boundary. Then, it is known by Alexandrov that $X$ is locally geodesically complete. In particular, $\Sigma_{x} X$ is also compact and geodesically complete for every $x \in X$. In this case, Proposition 3.1 in [8] shows that $\Sigma_{x} X$ is homeomorphic to 
$\mathbb{S}^{1}$. Therefore, we see that $X$ is locally bi-Lipschitz homeomorphic to $\mathbb{R}^{2}$. Namely, $X$ is a 2-dimensional Lipschitz manifold.

For $0<t<T$, we denote by $A_{x}(T, t ; X):=B_{x}(T ; X) \backslash \bar{B}_{x}(t ; X)$ the metric annulus around $x$.

In this paper, we show Proposition 3.1 by applying Theorem 2.3 adequately since $C_{x} X$ is of 2-dimension:

Proof of Proposition 3.1. Now, we note that every point in $C_{x} X \backslash\{\star\}$ has the space of directions isometric to $\mathbb{S}^{1}(1)$ since $\Sigma_{x} X$ is a circle, Also, $B_{\star}\left(1 ; C_{x} X\right)$ is an open 2-disk. We consider the (topological) annulus $A_{\star}\left(1,1 / 2 ; C_{x} X\right)$.

Since $\bar{B}_{x}(1 ; \lambda X)$ converges to $\bar{B}_{\star}\left(1 ; C_{x} X\right)$ as $\lambda \nearrow \infty$, we obtain the following: For any $\epsilon>0$, we have a sufficiently large number $J \gg 1$ such that, for each $j \in \mathbb{N} \cup\{0\}, d_{G H}\left(\bar{B}_{x}\left(1 ; 2^{J+j} X\right), \bar{B}_{\star}\left(1 ; C_{x} X\right)\right)<\epsilon$. Considering $A_{\star}\left(1,1 / 2 ; C_{x} X\right)$, we obtain the following from the arguments discussed in [15] (cf. Theorem 2.3, Remark 2.4):

Claim 3.5. For each $j$, we have a $\vartheta(\epsilon)$-almost isometry

$$
\widehat{h}_{j}: 2^{J+j} X \supset \widehat{U}_{j} \rightarrow A_{\star}\left(1,1 / 2 ; C_{x} X\right)
$$

for some open set $\widehat{U}_{j}$ satisfying:

$$
\begin{aligned}
A_{x}\left(1-\vartheta(\epsilon),(1 / 2)+\vartheta(\epsilon) ; 2^{J+j} X\right) & \subset \widehat{U}_{j} \\
& \subset A_{x}\left(1+\vartheta(\epsilon),(1 / 2)-\vartheta(\epsilon) ; 2^{J+j} X\right)
\end{aligned}
$$

Hence, by Claim 3.5, we obtain a homeomorphism

$$
h_{j}: X \supset U_{j}:=\left(1 / 2^{J+j}\right) \widehat{U}_{j} \rightarrow A_{\mathbf{o}}\left(1 / 2^{J+j}, 1 / 2^{J+j+2} ; \mathbb{R}^{2}\right),
$$

where $\mathbf{o} \in \mathbb{R}^{2}$ is the origin.

Next, assume that we have a homeomorphism

$$
H_{j}: \bigcup_{k=0}^{j} U_{k} \rightarrow A_{\mathbf{o}}\left(1 / 2^{J}, 1 / 2^{J+j+2} ; \mathbb{R}^{2}\right) .
$$

Then, using $h_{j+1}$, we can construct a homeomorphism

$$
H_{j+1}: \bigcup_{k=0}^{j+1} U_{k} \rightarrow A_{\mathbf{o}}\left(1 / 2^{J}, 1 / 2^{J+j+3} ; \mathbb{R}^{2}\right)
$$

such that $\left.H_{j+1}\right|_{\cup_{k=0}^{j} U_{k}}=H_{j}$, which is guaranteed by (3.1). Hence, we can define the map

$$
H_{\infty}: B_{x}\left(1 / 2^{J} ; X\right)=\bigcup_{k=0}^{\infty} U_{k} \rightarrow B_{\mathbf{o}}\left(1 / 2^{J} ; \mathbb{R}^{2}\right)
$$


with $H_{\infty}(x):=\mathbf{o}$ such that $\left.H_{\infty}\right|_{\cup_{k=0}^{j} U_{k}}=H_{j}, j=0, \ldots, \infty$. Then, $H_{\infty}$ is a homeomorphism, which completes the Proof of Proposition 3.1.

Now, for a given point, we provide the following local volume growth condition to ensure a 2-disk neighborhood, which is a generalization of Proposition C:

Proposition 3.6. For $\kappa \in \mathbb{R}$, let us denote by $X$ a locally compact, geodesically complete Alexandrov space with curvature $\leq \kappa$. Assume that a point $x \in \bar{X}^{2}$ satisfies the following:

$$
\mathcal{H}^{2}\left(B_{x}(T ; X)\right) / \omega_{\kappa}^{2}(T)<3 / 2
$$

for some $T \in\left(0, \operatorname{Comp}_{\kappa} \operatorname{Rad}(x)\right]$. Then, we have a positive number $t=$ $t_{x}>0$ such that $B_{x}(t ; X)$ is homeomorphic to a 2-dimensional open disk $B^{2} \subset \mathbb{R}^{2}$.

Remark 3.7. The above local volume growth condition (3.2) is optimal for Proposition 3.6: Actually, consider $X=\mathbb{S}^{2}(1) \sqcup \mathbb{H}^{2}(1)$ / equator, as in Example 1.1, which is a CAT(1)-space. Then, every point $x \in \bar{X}^{2}$ in the attached equator satisfies $\mathcal{H}^{2}\left(B_{x}(T ; X)\right) / \omega_{1}^{2}(T)=3 / 2$ for any $T \in(0, \pi]$, and $x$ never possess a neighborhood homeomorphic to a 2 -disk.

Proof of Proposition 3.6. By Proposition 2.9 and the assumption (3.2), we have

$$
\frac{\mathcal{H}^{n}\left(B_{x}(t ; X)\right)}{\omega_{1}^{n}(t)} \leq \frac{\mathcal{H}^{n}\left(B_{x}(T ; X)\right)}{\omega_{1}^{n}(T)}<\frac{3}{2}
$$

for any $t \in(0, T], n=2$. It then follows from Lemma 2.7 and (2.10) that

$$
\frac{3}{2}>\frac{\mathcal{H}^{n}\left(B_{x}(t ; X)\right)}{\omega_{1}^{n}(t)} \rightarrow \frac{\mathcal{H}^{n-1}\left(\Sigma_{x} X\right)}{\mathcal{H}^{n-1}\left(\mathbb{S}^{n-1}(1)\right)} \geq 1
$$

as $t \searrow 0$. Now, note that $\Sigma_{x} X={\overline{\left(\Sigma_{x} X\right)}}^{1}$ by Lemma 2.2.

Next, we investigate the following 1-dimensional case:

Proposition 3.8. Let $X$ be a compact, geodesically complete CAT(1)-space satisfying $X=\bar{X}^{1}$ and

$$
\mathcal{H}^{1}(X)<(3 / 2) \mathcal{H}^{1}\left(\mathbb{S}^{1}(1)\right) .
$$

Then, $X$ is homeomorphic to $\mathbb{S}^{1}$.

Proof. Since $X=\bar{X}^{1}$, we see that $X$ has a structure of finite graph equipped with the vertex set containing $S_{\pi}(X)$ (cf. Lemma 2.9 in [14]).

Suppose that $S_{\pi}(X) \neq \emptyset$. Taking $x \in S_{\pi}(X)$, we have (at least three) minimizing geodesics $\gamma_{x, i}:[0, \pi] \rightarrow X, i=1,2,3$, with $\gamma_{x, i}(0)=x$ such that $\gamma_{x, i}((0, \pi)) \cap \gamma_{x, j}((0, \pi))=\emptyset$ for $i \neq j$ since $\operatorname{Inj} \operatorname{Rad}(x) \geq \pi$. This implies 
that $\mathcal{H}^{1}(X) \geq(3 / 2) \mathcal{H}^{1}\left(\mathbb{S}^{1}(1)\right)$, which contradicts to the present assumption. Hence, $S_{\pi}(X)=\emptyset$, and hence $X$ is homeomorphic to $\mathbb{S}^{1}$.

Recall that the point $x \in \bar{X}^{2}$ in Proposition 3.6 has the space of directions $\Sigma_{x} X$ which is a CAT(1)-space as researched in Proposition 3.8. Therefore, Propositions 3.1 and 3.8 conclude Proposition 3.6.

Thus, we have shown Proposition C.

\section{A sphere theorem for 2-dimensional CAT(1)-spaces.}

In this section, we prove Theorems A and B.

4.1. Proof of Theorem A. First, we observe the following metric properties:

Lemma 4.1. For given $n \in \mathbb{N}$, let $X$ be a compact, geodesically complete CAT(1)-space with $X=\bar{X}^{n}$ such that $\mathcal{H}^{n}(X)<(3 / 2) \mathcal{H}^{n}\left(\mathbb{S}^{n}(1)\right)$. Then, the following hold:

(i) For any $x \in X$, we have $\mathcal{H}^{n-1}\left(\Sigma_{x} X\right)<(3 / 2) \mathcal{H}^{n-1}\left(\mathbb{S}^{n-1}(1)\right)$.

(ii) For $z_{1}, z_{2} \in X$ with $d_{X}\left(z_{1}, z_{2}\right)=\operatorname{diam} X$, we obtain

$$
X=B_{z_{1}}(\pi ; X) \cup B_{z_{2}}(\pi ; X) .
$$

Proof. (i): Now, (i) follows from the similar argument as that discussed in the Proof of Proposition 3.6.

(ii): Suppose that, $X \neq B_{z_{1}}(\pi ; X) \cup B_{z_{2}}(\pi ; X)$, i.e., we have a point $z_{3} \in X$ satisfying $d_{X}\left(z_{i}, z_{3}\right) \geq \pi, i=1,2$. Then, since $X$ is geodesically complete, we have $d_{X}\left(z_{1}, z_{2}\right)=\operatorname{diam} X \geq \pi$. Hence, we obtain $B_{z_{i}}(\pi / 2 ; X) \cap$ $B_{z_{j}}(\pi / 2 ; X)=\emptyset$ for $i \neq j, i, j=1,2,3$. Then, by Proposition 2.6, we have

$$
\begin{aligned}
\mathcal{H}^{n}(X) & \geq \mathcal{H}^{n}\left(\bigsqcup_{i=1}^{3} B_{z_{i}}(\pi / 2 ; X)\right)=\sum_{i=1}^{3} \mathcal{H}^{n}\left(B_{z_{i}}(\pi / 2 ; X)\right) \\
& \geq 3 \omega_{1}^{n}(\pi / 2)=(3 / 2) \mathcal{H}^{n}\left(\mathbb{S}^{n}(1)\right) .
\end{aligned}
$$

This contradicts to the present assumption $\mathcal{H}^{n}(X)<(3 / 2) \mathcal{H}^{n}\left(\mathbb{S}^{n}(1)\right)$, which proves (ii).

Here, we prove Theorem A:

Proof of Theorem A. Let $X$ denote a compact, geodesically complete CAT(1)space satisfying $X=\bar{X}^{2}$ and $\mathcal{H}^{2}(X)<(3 / 2) \mathcal{H}^{2}\left(\mathbb{S}^{2}(1)\right)$. Then, by Proposition 3.6 and Lemma 4.1.(i), $X$ is a 2-dimensional topological manifold.

Now, we note that $X$ can be covered by two contractible open sets by Lemma 4.1.(ii). Hence, since $X$ is 2-dimensional, we obtain

$$
X=B_{1}^{2} \cup B_{2}^{2}
$$


for some open 2-disks $B_{1}^{2}, B_{2}^{2}$. Then, the Jordan curve theorem concludes that $X$ is homeomorphic to $\mathbb{S}^{2}$.

In this way, we have completed the Proof of Theorem A.

4.2. Proof of Theorem B. First, we study the 1-dimensional case:

Proposition 4.2. Let $X$ be a compact, geodesically complete CAT(1)-space with $X=\bar{X}^{1}$ such that $\mathcal{H}^{1}(X)=(3 / 2) \mathcal{H}^{1}\left(\mathbb{S}^{1}(1)\right)$. Then, $X$ is either a circle or isometric to $\mathbb{S}^{1}(1) \sqcup \mathbb{H} \mathbb{S}^{1}(1)$ / equator .

Then, we again recall that $X$ has a structure of finite graph equipped with the vertex set containing $S_{\pi}(X)$ since $X=\bar{X}^{1}$.

Here, let $\mathbb{S}^{1}(1) \sqcup[0, \pi] / p=\{0\}, \hat{p}=\{\pi\}$ denote the CAT(1)-space as in Example 1.1.(ii). Then, note that $\mathbb{S}^{1}(1) \sqcup \mathbb{H} \mathbb{S}^{1}(1) /$ equator is isometric to $\mathbb{S}^{1}(1) \sqcup$ $[0, \pi] / p=\{0\}, \hat{p}=\{\pi\}$.

Proof of Proposition 4.2. Let us denote by $\left\{z_{i}\right\}$ a maximal $\pi$-discrete set in $X$, i.e., $d_{X}\left(z_{i}, z_{j}\right) \geq \pi$ for $i \neq j$.

First, note that $\sharp\left\{z_{i}\right\} \geq 2$ for any maximal $\pi$-discrete set $\left\{z_{i}\right\} \subset X$ since $X$ is geodesically complete. On the other hand, from Proposition 2.6 and the present assumption $\mathcal{H}^{1}(X)=(3 / 2) \mathcal{H}^{1}\left(\mathbb{S}^{1}(1)\right)$, we can conclude that $\sharp\left\{z_{i}\right\} \leq 3$ for any such $\left\{z_{i}\right\} \subset X$.

Claim 4.3. Let $X$ be as in Proposition 4.2. If $\sharp\left\{z_{i}\right\}=2$ for any maximal $\pi$-discrete set $\left\{z_{i}\right\} \subset X$, then $X$ is a circle.

Proof. Take a maximal $\pi$-discrete set $\left\{z_{1}, z_{2}\right\} \subset X$. It then follows from the maximality of $\left\{z_{1}, z_{2}\right\} \subset X$ that $X=B_{z_{1}}(\pi ; X) \cup B_{z_{2}}(\pi ; X)$.

Suppose that some point $x \in X$ is contained in $S_{\pi}(X)$, i.e., $x$ is the vertex of $X$. Then, $\operatorname{since} \operatorname{Inj} \operatorname{Rad}(x) \geq \pi$, we have at least three minimizing geodesics $\gamma_{x, k}:[0, \pi] \rightarrow X, k=1,2,3$, with $\gamma_{x, k}(0)=x$ such that $\gamma_{x, k}((0, \pi)) \cap \gamma_{x, l}((0, \pi))=\emptyset$ for $k \neq l$. Hence, we obtain a $\pi$-discrete set $\left\{\gamma_{x, k}(\pi / 2)\right\}_{k=1,2,3} \subset X$. This is a contradiction to the assumption in Claim 4.3. Hence, $X$ is a circle.

Claim 4.4. Let $X$ be as in Proposition 4.2. If $\sharp\left\{z_{i}\right\}=3$ for some maximal $\pi$-discrete set $\left\{z_{i}\right\} \subset X$, then $X$ is isometric to the CAT(1)-space $\mathbb{S}^{1}(1) \sqcup$ $\mathbb{H} \mathbb{S}^{1}(1) /$ equator.

Proof. In this case, by Proposition 2.6 and the present volume assumption $\mathcal{H}^{1}(X)=(3 / 2) \mathcal{H}^{1}\left(\mathbb{S}^{1}(1)\right)$, we see that $\bar{B}_{z_{i}}(\pi / 2 ; X)$ is isometric to $[0, \pi]$ for each $i=1,2,3$. Considering how $\bar{B}_{z_{i}}(\pi / 2 ; X), i=1,2,3$, meet each other, we can show that $X=\mathbb{S}^{1}(1) \sqcup \mathbb{H} \mathbb{S}^{1}(1) /$ equator .

Therefore, Claims 4.3 and 4.4 conclude Proposition 4.2. 
Here, let us begin proving Theorem B:

Proof of Theorem B. For a while, we denote by $X$, as in Theorem B, a compact, geodesically complete CAT(1)-space with $X=\bar{X}^{2}$ such that $\mathcal{H}^{2}(X)=(3 / 2) \mathcal{H}^{2}\left(\mathbb{S}^{2}(1)\right)$. Then, similarly to the Proof of Proposition 4.2, we see that $\sharp\left\{z_{i}\right\}=2$ or 3 for any maximal $\pi$-discrete set $\left\{z_{i}\right\} \subset X$ from Proposition 2.6 and the present assumption $\mathcal{H}^{2}(X)=(3 / 2) \mathcal{H}^{2}\left(\mathbb{S}^{2}(1)\right)$. We now note that, for any $x \in X$, the space of directions $\Sigma_{x} X$ is either a circle or isometric to $\mathbb{S}^{1}(1) \sqcup \mathbb{H} \mathbb{S}^{1}(1)$ / equator by Lemma 2.2 , (2.2), Propositions 3.8 and 4.2 .

Lemma 4.5. Let $X$ be as in Theorem B. If $\sharp\left\{z_{i}\right\}=2$ for any maximal $\pi$-discrete set $\left\{z_{i}\right\} \subset X$, then $X$ is homeomorphic to $\mathbb{S}^{2}$.

Proof. For a maximal $\pi$-discrete set $\left\{z_{1}, z_{2}\right\} \subset X$, we have

$$
X=B_{z_{1}}(\pi ; X) \cup B_{z_{2}}(\pi ; X)
$$

from the maximality of $\left\{z_{1}, z_{2}\right\} \subset X$.

We next show that $\Sigma_{x} X$ is a circle for every $x \in X$. Suppose that $\Sigma_{x} X$ is isometric to $\mathbb{S}^{1}(1) \sqcup \mathbb{H} \mathbb{S}^{1}(1) /$ equator, and hence, isometric to $\mathbb{S}^{1}(1) \sqcup$

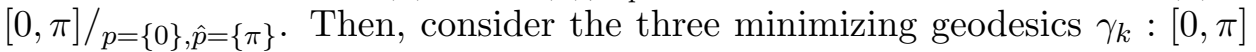
$\rightarrow \Sigma_{x} X, k=1,2,3$, such that $\gamma_{k}(0)=p, \gamma_{k}(\pi)=\hat{p}$, and that $\gamma_{k}((0, \pi)) \cap$ $\gamma_{l}((0, \pi))=\emptyset$ for $k \neq l$. We now take the direction $v_{k}:=\gamma_{k}(\pi / 2) \in \Sigma_{x} X$, and a point $y_{k} \in X$ satisfying $v_{x y_{k}}=v_{k} \in \Sigma_{x} X$ and $d_{X}\left(x, y_{k}\right)=\pi / 2$. Since $\angle_{x}\left(y_{k}, y_{l}\right)=\pi$ for $k \neq l$ in this case, $\left\{y_{k}\right\}_{k=1,2,3} \subset X$ forms a $\pi$-discrete set in $X$. This is a contradiction to the assumption in Lemma 4.5. Hence, $\Sigma_{x} X$ is a circle for every $x \in X$.

Therefore, by Proposition 3.1, the space $X$ is a 2-dimensional topological manifold. Similarly to the Proof of Theorem A, we can show that $X$ is homeomorphic to $\mathbb{S}^{2}$.

Lemma 4.6. Let $X$ be as in Theorem B. If $\sharp\left\{z_{i}\right\}=3$ for some maximal $\pi$-discrete set $\left\{z_{i}\right\} \subset X$, then $X$ is either homeomorphic to $\mathbb{S}^{2}$ or isometric to $\mathbb{S}^{2}(1) \sqcup \mathbb{H} \mathbb{S}^{2}(1) /$ equator.

Proof of Lemma 4.6. In this case, by Proposition 2.6 and the assumption $\mathcal{H}^{2}(X)=(3 / 2) \mathcal{H}^{2}\left(\mathbb{S}^{2}(1)\right)$, we also see that $\bar{B}_{z_{i}}(\pi / 2 ; X)$ is isometric to $\mathbb{H} \mathbb{S}^{2}(1)$ for each $i=1,2,3$, and that $X=\cup\left\{\bar{B}_{z_{i}}(\pi / 2 ; X) \mid i=1,2,3\right\}$.

Next, we observe how $\bar{B}_{z_{i}}(\pi / 2 ; X), i=1,2,3$, meet each other along their boundaries $\mathbb{S}_{i}^{1}(1):=\partial B_{z_{i}}(\pi / 2 ; X)$.

Claim 4.7. $\bar{B}_{z_{i}}(\pi / 2 ; X) \cap \bar{B}_{z_{j}}(\pi / 2 ; X)=\mathbb{S}_{i}^{1}(1) \cap \mathbb{S}_{j}^{1}(1)$ is a nonempty subset of $X$ for each $i \neq j$.

Proof. We here only verify that $\bar{B}_{z_{i}}(\pi / 2 ; X) \cap \bar{B}_{z_{j}}(\pi / 2 ; X) \neq \emptyset$ for each $i \neq j$. 
Suppose that $\bar{B}_{z_{i}}(\pi / 2 ; X) \cap \bar{B}_{z_{j}}(\pi / 2 ; X)=\emptyset$ for some $i \neq j$. Then, for such $i$, we have $\bar{B}_{z_{i}}(\pi / 2 ; X) \cap \bar{B}_{z_{k}}(\pi / 2 ; X) \neq \emptyset$, in particular, we see that $\bar{B}_{z_{i}}(\pi / 2 ; X) \cap \bar{B}_{z_{k}}(\pi / 2 ; X)$ is a closed, convex subset isometric to $\mathbb{S}^{1}(1)$ since $X$ is geodesically complete.

On the other hand, for such $j$, the set $\bar{B}_{z_{j}}(\pi / 2 ; X) \cap \bar{B}_{z_{k}}(\pi / 2 ; X)$ is also a closed, convex subset isometric to $\mathbb{S}^{1}(1)$, which yields a contradiction.

Now, the connected finite graph $\cup \mathbb{S}_{i}^{1}(1)=\cup\left\{\mathbb{S}_{i}^{1}(1) \mid i=1,2,3\right\}$ equips the interior distance $d_{X}$ because $\mathbb{S}_{i}^{1}(1)$ is isometrically embedded in $X$. Hence, the injectivity radius of $\cup \mathbb{S}_{i}^{1}(1)$ is not smaller than $\pi$.

Furthermore, the diameter of $\cup \mathbb{S}_{i}^{1}(1)$ is equal to $\pi$ : Actually, we only verify the following essential case: Some points $x_{i} \in \mathbb{S}_{i}^{1}(1)$ and $x_{j} \in \mathbb{S}_{j}^{1}(1)$ satisfy $x_{i} \notin \mathbb{S}_{j}^{1}(1)$ and $x_{j} \notin \mathbb{S}_{i}^{1}(1)$. Then, by Claim 4.7 , we have $x_{i}, x_{j} \in \mathbb{S}_{k}^{1}(1)$, and hence $d_{X}\left(x_{i}, x_{j}\right) \leq \pi$.

It is seen by Lemma 6.1 in [4] (cf. [6]) that such a graph $\cup \mathbb{S}_{i}^{1}(1)$ is isometric to either $\mathbb{S}^{1}(1)$ or $\mathbb{S}^{1}(1) \sqcup \mathbb{H} \mathbb{S}^{1}(1) /$ equator.

If $\cup \mathbb{S}_{i}^{1}(1)=\mathbb{S}^{1}(1)$, then $X$ is isometric to $\mathbb{S}^{2}(1) \sqcup \mathbb{H}^{2}(1) /$ equator. If $\cup \mathbb{S}_{i}^{1}(1)=\mathbb{S}^{1}(1) \sqcup \mathbb{H} \mathbb{S}^{1}(1) /$ equator, then $X$ is homeomorphic to $\mathbb{S}^{2}$. This completes the Proof of Lemma 4.6.

Thereby, we have proved Theorem B.

\section{Topological embeddings of CAT $(\kappa)$-spaces of dimension not greater than 2.}

\subsection{On the local structure of 2-dimensional spaces with curvature} bounded above. The local structure of spaces with curvature bounded above has been studied by Burago and Buyalo [8], Kleiner [12], and others. We here observe the local structure of spaces of the Hausdorff dimension not greater than 2 .

Let us denote by $X$ a locally compact, geodesically complete Alexandrov space with curvature $\leq \kappa$ satisfying $X=\widehat{X}^{2}$. Then, we obtain the following:

Proposition 5.1. $X=\bar{X}^{2} \cup \bar{X}^{1} \cup \bar{X}^{0}$. In particular, $\bar{X}^{2}, \bar{X}^{0}$ are closed, and $\bar{X}^{1}$ is open in $X$.

A direction $v \in \Sigma_{x} X$ is said to be isolated if $\angle_{x}(u, v)=\pi$ for any $u \in \Sigma_{x} X$. In this case, the subset $\{v\}$ itself is a connected component of $\Sigma_{x} X$.

To show Proposition 5.1, we first study isolated directions:

Lemma 5.2. Let $x \in X$ be a point possessing an isolated direction $v \in$ $\Sigma_{x} X$. Then, we have a positive number $\bar{t}=\bar{t}_{x, v}>0$ satisfying the following: If $\gamma_{1}$ and $\gamma_{2}$ are minimizing geodesics emanating from $x$ directed by $v$, then $\gamma_{1}(t)=\gamma_{2}(t)$ holds for any $t \in(0, \bar{t})$. 
Proof. Suppose that this claim is not true. We may now assume the following: There exist $y_{i}, z_{i}, w_{i} \in X \backslash\{x\}$ with $y_{i}, z_{i}, w_{i} \rightarrow x$ such that:

(i) $t_{i}:=d_{X}\left(x, y_{i}\right)=d_{X}\left(x, z_{i}\right)$,

(ii) $y_{i} \in x w_{i}, y_{i} \neq z_{i}$,

(iii) $v=v_{x y_{i}}=v_{x z_{i}}=v_{x w_{i}} \in \Sigma_{x} X$.

Let $p_{i} \in X$ be a point with $z_{i} \in y_{i} p_{i}$ so that $d_{X}\left(y_{i}, p_{i}\right)$ is uniformly constant. Then, we may assume that there is $p_{0} \neq x$ satisfying $p_{i} \rightarrow p_{0}$, $x p_{i} \rightarrow x p_{0}$, and $v_{x p_{i}} \rightarrow v_{x p_{0}} \in \Sigma_{x} X$. Since $\angle_{x}\left(y_{i}, z_{i}\right)=0$, the inequality $\angle_{y_{i}}\left(w_{i}, p_{i}\right) \geq \pi / 2-\vartheta\left(t_{i}\right)$ follows from comparison geometry. Because $v \in$ $\Sigma_{x} X$ is isolated, we have $\angle_{x}\left(y_{i}, p_{0}\right)=\pi$ from the upper semi-continuity of angles. The choice of $p_{i}, p_{0}$ implies that $v_{x p_{i}}, v_{x p_{0}}$ are uniformly contained in the same connected component of $\Sigma_{x} X$, which also implies $\angle_{x}\left(y_{i}, p_{i}\right)=\pi$. Since $x \notin y_{i} p_{i}$, we obtain a contradiction.

Remark 5.3. Lemma 5.2 also holds independently of the assumption $X=$ $\widehat{X}^{2}$.

Proof of Proposition 5.1. Let us consider the essential case $\bar{X}^{0}=\emptyset$. Assume that we have a point $x \in X$ with $x \notin \bar{X}^{2}$. Then, since $X=\widehat{X}^{2}$, there exists $t>0$ such that $\mathcal{H}^{2}\left(B_{x}(t ; X)\right)=0$. Because of the existence of the Lipschitz onto map

$$
\log _{x}: B_{x}(t ; X) \ni y \mapsto\left(v_{x y}, d_{X}(x, y)\right) \in B_{\star}\left(t ; C_{x} X\right)
$$

$\left(\log _{x}(x):=\star\right)$, we have $\mathcal{H}^{1}\left(\Sigma_{x} X\right)=0$. This implies that $\Sigma_{x} X$ is composed of at most finitely many isolated points. Hence, by Lemma 5.2, we have $x \in \bar{X}^{1}$.

Furthermore, it is known by [15] that $\bar{X}^{2}$ is closed. Therefore, we obtain Proposition 5.1.

Next, we investigate the 2-dimensional part. Let us define

$$
\begin{aligned}
& R_{x}^{2}(t):=\left\{y \in X \mid y \in \bar{X}^{2}, d_{X}(x, y)<t\right\}, \\
& \bar{R}_{x}^{2}(t):=\left\{y \in X \backslash\{x\} \mid v_{x y} \in{\overline{\left(\Sigma_{x} X\right)}}^{1}, d_{X}(x, y)<t\right\} \cup\{x\} .
\end{aligned}
$$

Lemma 5.4. For any $x \in \bar{X}^{2}$, there exists a positive number $t_{x}>0$ such that $R_{x}^{2}(t)=\bar{R}_{x}^{2}(t)$ for any $t \in\left(0, t_{x}\right)$.

Proof. To show $R_{x}^{2}(t) \subset \bar{R}_{x}^{2}(t)$, suppose that we have some points $y_{i}, i=$ $1,2, \ldots$, converging to $x$ with $y_{i} \in \bar{X}^{2}$ such that $v_{x y_{i}} \in \Sigma_{x} X$ are isolated. Since $\Sigma_{x} X$ is compact, we may assume $v_{x y_{i}}=v$ for some isolated direction $v$. Then, Lemma 5.2 yields that $y_{i} \in \bar{X}^{1}$ for any sufficiently large $i$, which is a contradiction.

On the other hand, suppose that we have a point $y \in \bar{R}_{x}^{2}(t)$ such that $y \notin \bar{X}^{2}$. Now, $y \in \bar{X}^{1}$ follows from Proposition 5.1. Then, the existence of 
$\log _{x}$ implies that $v_{x y}$ is isolated, which is a contradiction. Hence, we obtain $\bar{R}_{x}^{2}(t) \subset R_{x}^{2}(t)$.

We here claim the local convexity of the 2-dimensional part.

Proposition 5.5. For any $x \in \bar{X}^{2}$, there exists $t_{x}>0$ such that $R_{x}^{2}(t)$ is convex in $X$ for any $t \in\left(0, t_{x}\right)$. In other words, $\bar{X}^{2}$ is locally convex in $X$.

Proof. Suppose this claim is not true, i.e., suppose that there exist points $y_{i}, z_{i} \in \bar{X}^{2}$ with $y_{i}, z_{i} \rightarrow x$ such that we have a point $w_{i} \in y_{i} z_{i} \cap \bar{X}^{1}$.

If $x \notin y_{i} z_{i}$ for infinitely many $i$, then $v_{x w_{i}}$ are isolated by Lemma 5.4. This implies that $\angle_{x}\left(y_{i}, w_{i}\right)=\pi$, which yields a contradiction.

If $x \in y_{i} z_{i}$ for infinitely many $i$, then without loss of generality we may assume that $w_{i} \neq x$ is contained in $x y_{i}$. Then, by Lemma 5.4, $v_{x y_{i}}=$ $v_{x w_{i}} \in{\overline{\left(\Sigma_{x} X\right)}}^{1}$, and hence $w_{i} \in \bar{X}^{2}$. This is a contradiction. We thus prove Proposition 5.5.

Remark 5.6. Let $x \in \bar{X}^{2}$ be a point such that the space of directions $\Sigma_{x} X$ is composed of a circle and finitely many points. Then, by Propositions 3.1 and 5.5, we completely understand the local topological structure around $x$. Namely, $R_{x}^{2}(t)$ is homeomorphic to $B^{2}$, and $B_{x}(t ; X)$ is composed of $R_{x}^{2}(t)$ and the finitely many minimizing geodesics emanating from $x$ directed by the isolated directions for sufficiently small $t>0$. This proposition can be also proved by the results of Kleiner, Burago and Buyalo [8].

\subsection{A topological embedding into CAT(1)-spaces of dimension}

$\leq \mathbf{2}$. Next, we prove the following which is a generalization of Theorem A:

Theorem 5.7. Let $X$ be a compact, geodesically complete CAT(1)-space with $X=\widehat{X}^{2}, \bar{X}^{2} \neq \emptyset$ satisfying $\mathcal{H}^{2}(X)<(3 / 2) \mathcal{H}^{2}\left(\mathbb{S}^{2}(1)\right)$. Then, the compact, locally convex subset $Y:=\bar{X}^{2} \subset X$ is a Lipschitz manifold homeomorphic to $\mathbb{S}^{2}$. Moreover, $Y$ is a compact, geodesically complete CAT(1)-space with respect to the interior distance in $Y$.

Proof. Now, we define $Y:=\bar{X}^{2}$. Then, $\Sigma_{x} X$ is composed of a circle and at most finitely many points for every $x \in Y$ from the assumption $\mathcal{H}^{2}(X)<$ $(3 / 2) \mathcal{H}^{2}\left(\mathbb{S}^{2}(1)\right)$ and the same argument as that discussed in Propositions 3.6 and 3.8. Hence, by Remark 5.6, $R_{x}^{2}(t)$ is homeomorphic to $B^{2}$, and $B_{x}(t ; X)$ is the union of $R_{x}^{2}(t)$ and the finitely many minimizing geodesics emanating from $x$ for sufficiently small $t>0$. Therefore, we see that $Y$ is a compact, 2-dimensional Lipschitz manifold without boundary.

Let us consider the interior distance $d_{Y}$ in $Y$ induced from $d_{X}$. Then, by Proposition 5.5, $d_{Y}$ locally coincides with $d_{X}$. Hence, $Y$ is an Alexandrov space with curvature $\leq 1$. Furthermore, for any $y_{1}, y_{2} \in Y$ with $d_{X}\left(y_{1}, y_{2}\right)<$ $\pi$, we have $d_{Y}\left(y_{1}, y_{2}\right)=d_{X}\left(y_{1}, y_{2}\right)$ from the CAT(1)-property of $X$. Since 
$\operatorname{Inj} \operatorname{Rad}(Y) \geq \pi$, we can show that $Y$ is a compact, geodesically complete CAT(1)-space with $Y=\bar{Y}^{2}$ such that $\mathcal{H}^{2}(Y)<(3 / 2) \mathcal{H}^{2}\left(\mathbb{S}^{2}(1)\right)$. Therefore, Theorem A implies Theorem 5.7.

Remark 5.8. The set $Y$ as that stated in Theorem 5.7 is not necessarily globally convex in $X$ since a minimizing geodesic in $X$ joining $y_{1}, y_{2} \in Y$ possibly passes through some 1-dimensional part. 


\section{Addendum from a topological view point}

From the preceding observation, it is perspective to be shown that:

Conjecture 6.1. For given positive integer $n \geq 3$, let $X$ be a compact, geodesically complete CAT(1)-space satisfying $X=\bar{X}^{n}$ and the following: $\mathcal{H}^{n}(X)<(3 / 2) \mathcal{H}^{n}\left(\mathbb{S}^{n}(1)\right)$. Then, $X$ is homeomorphic to $\mathbb{S}^{n}$.

The author does not know an example of $X$ as in the assumption in 6.1, which is not homeomorphic to $\mathbb{S}^{n}$.

Actually, by the arguments discussed above and the generalized Schoenflies theorem (cf. [21]), we can show the following which has been essentially proved by Coghlan and Itokawa [9]:

Theorem 6.2. Let $M$ be a compact, smooth Riemannian manifold of dimension $n$ which is also a CAT(1)-space. Assume that the following holds: $\mathcal{H}^{n}(M)<(3 / 2) \mathcal{H}^{n}\left(\mathbb{S}^{n}(1)\right)$. Then, $M$ is homeomorphic to $\mathbb{S}^{n}$.

Also, in the previous section, Proposition 3.1 plays an important role to study spaces with curvature bounded above from a topological view point. As a natural question, we provide:

Conjecture 6.3. Let $x \in X$ be a point in a locally compact, geodesically complete Alexandrov space with curvature $\leq \kappa$ such that $\Sigma_{x} X$ is homeomorphic to $\mathbb{S}^{n-1}$ for given $n \geq 3$. Then, $x$ has a neighborhood homeomorphic to some $n$-dimensional open disk.

The essential part of the problem in 6.3 is to observe singular points with serious singularities because of Theorem 3.1 in [8].

For finite dimensional Alexandrov spaces with curvature bounded below, it is known that the proposition as in 6.3 is affirmative from Perelman's stability theorem $([\mathbf{1 8}])$ : For a given space, if the other space of the same dimension is sufficiently close to it with respect to $d_{G H}$, then they are homeomorphic.

Our problem in 6.3 is different from that of the stability theorem. Kleiner ([12]) points out that, in general, the stability theorem does not hold for locally compact, geodesically complete spaces with curvature bounded above (cf. Example 2.7 in [14]).

In fact, for an arbitrary $\epsilon>0$, we can construct an example of compact, geodesically complete CAT(1)-space $X_{\epsilon}$ with $X_{\epsilon}=\bar{X}_{\epsilon}^{2}$ satisfying the following:

(i) $\mathcal{H}^{2}\left(X_{\epsilon}\right) \in\left(2 \mathcal{H}^{2}\left(\mathbb{S}^{2}(1)\right), 2 \mathcal{H}^{2}\left(\mathbb{S}^{2}(1)\right)+\epsilon\right)$.

(ii) $X_{\epsilon}$ admits no triangulation.

(iii) $X_{\epsilon}$ converges to $\mathbb{S}^{2}(1) \sqcup \mathbb{S}^{2}(1)$ / equator with respect to $d_{G H}$ as $\epsilon \rightarrow 0$.

Here, $\mathbb{S}^{2}(1) \sqcup \mathbb{S}^{2}(1) /$ equator denotes the quotient space obtained by gluing $\mathbb{S}^{2}(1)$ and $\mathbb{S}^{2}(1)$ along their equators. This example $X_{\epsilon}$ can be constructed 
by the similar way to that stated in Example 2.7 in [14]. Roughly speaking, the construction of $X_{\epsilon}$ is as follows:

First, we construct a region $C_{\epsilon} \subset \mathbb{R}^{2}$ as in Figure 3 , composed of a sequence of quadrangles whose size tend to 0 , surrounded by two piecewise broken curves $c_{\epsilon}$ and $\bar{c}_{\epsilon}$ joining $p_{\epsilon}$ and the limit point $\widehat{p}_{\epsilon}$, such that the lengths of $c_{\epsilon}$ and $\bar{c}_{\epsilon}$ is not greater than $\pi$, and that the area of $C_{\epsilon}$ is bounded above by $\vartheta(\epsilon)$.

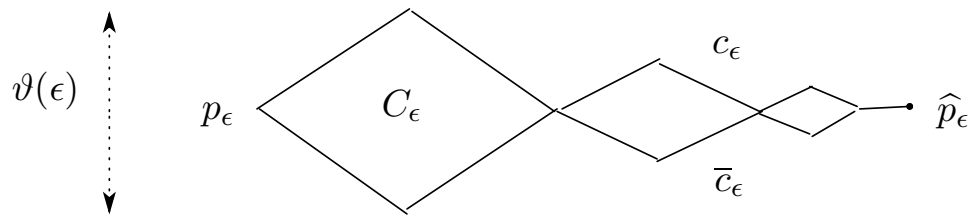

Figure 3. A region $C_{\epsilon} \subset \mathbb{R}^{2}$.

Next, we prepare a region $W_{\epsilon}^{1} \subset \mathbb{S}^{2}(1)=\mathbb{H}^{2}(1) \sqcup \mathbb{H} \mathbb{S}^{2}(1) /$ equator as in Figure 4 with its boundary $\partial W_{\epsilon}^{1}$ such that:

(i) $\mathbb{H}^{2}(1)$ is a proper subset of $W_{\epsilon}^{1}$.

(ii) The area of $W_{\epsilon}^{1} \backslash \mathbb{H} \mathbb{S}^{2}(1)$ is bounded above by $\vartheta(\epsilon)$.

(iii) Let us also prepare another three regions $W_{\epsilon}^{i}, i=2,3,4$, isometric to $W_{\epsilon}^{1}$. If we choose an appreciate subarc $\tau_{\epsilon}^{i}(i=1,2,3,4)$ of $\partial W_{\epsilon}^{i}$, then the quotient space $X_{\epsilon}:=C_{\epsilon} \sqcup\left(\sqcup_{i=1}^{4} W_{\epsilon}^{i}\right) / \sim$ made by the relations $\tau_{\epsilon}^{1}=c_{\epsilon}=\tau_{\epsilon}^{2}$ and $\tau_{\epsilon}^{3}=\bar{c}_{\epsilon}=\tau_{\epsilon}^{4}$ is a compact, geodesically complete CAT(1)-space.

To realize this, we must be careful of the "geodesic curvature" (in a generalized sense) of $c_{\epsilon}, \bar{c}_{\epsilon}, \tau_{\epsilon}^{i}$, and $\partial W_{\epsilon}^{i}$.
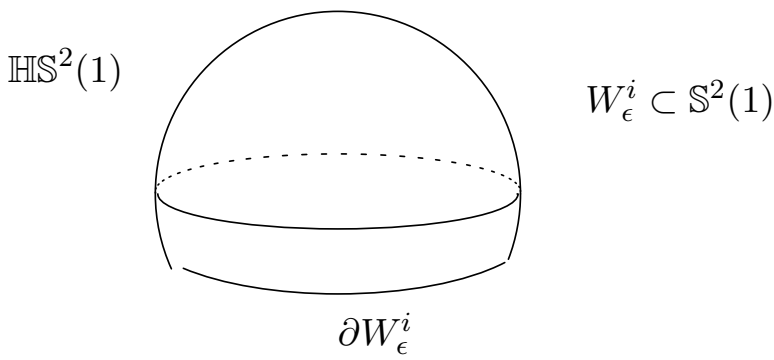

Figure 4. A region $W_{\epsilon}^{i} \subset \mathbb{S}^{2}(1)$

In this way, we can obtain such a wild example $X_{\epsilon}$ which admits no triangulation around $\widehat{p}_{\epsilon} \in C_{\epsilon} \subset X_{\epsilon}$. Furthermore, its construction implies that $X_{\epsilon}$ converges to $\mathbb{S}^{2}(1) \sqcup \mathbb{S}^{2}(1) /$ equator with respect to $d_{G H}$ as $\epsilon \rightarrow 0$, and then, $\mathcal{H}^{2}\left(X_{\epsilon}\right) \rightarrow \mathcal{H}^{2}\left(\mathbb{S}^{2}(1) \sqcup \mathbb{S}^{2}(1) /\right.$ equator $)$.

We hence mention that the following problem is still open: 
Problem 6.4. Describe the homeomorphism type of a given, compact, geodesically complete CAT(1)-space $X$ satisfying $X=\bar{X}^{2}$ and

$$
\mathcal{H}^{2}(X) \in\left((3 / 2) \mathcal{H}^{2}\left(\mathbb{S}^{2}(1)\right), 2 \mathcal{H}^{2}\left(\mathbb{S}^{2}(1)\right)\right] .
$$

On the other hand, we can observe the number of the homotopy types of such CAT(1)-spaces. We now discuss it more generally as follows:

For given constants $\kappa \in \mathbb{R}, n \in \mathbb{N}, V>0$, and $R>0$, let us denote by $\mathcal{A}(\kappa, n, V, R)$ the isometry classes of all compact, geodesically complete Alexandrov spaces with curvature $\leq \kappa$ such that $X=\bar{X}^{n}, \mathcal{H}^{n}(X) \leq V$, and that $\mathrm{CAT}_{\kappa} \operatorname{Rad}(x) \geq R$ for every $x \in X$.

For $X \in \mathcal{A}(\kappa, n, V, R)$, the compactness of $X$ and the condition that $\operatorname{CAT}_{\kappa} \operatorname{Rad}(x) \geq R$ for every $x \in X$ guarantee the following ([20], cf. Lemma I.7A.15 in [7]): $X$ is homotopy equivalent to a finite Euclidean simplicial complex $K$ which is the nerve obtained by a finite covering

$$
\mathcal{U}=\left\{B_{x_{i}}(R / 10 ; X) \mid i \in I_{X}\right\}
$$

of $X$ such that $\left\{x_{i}\right\}_{i \in I_{X}}$ is a maximal $(R / 20)$-discrete set in $X$.

Now, by Proposition 2.6, the number of its covering $I_{X}$ is bounded above by a constant depending only on $\kappa, n, V$, and $R$. Therefore, we have the following:

Proposition 6.5. For given constants $\kappa \in \mathbb{R}, n \in \mathbb{N}, V>0$, and $R>0$, the number of the homotopy types of $\mathcal{A}(\kappa, n, V, R)$ is bounded above by a constant depending only on $\kappa, n, V$, and $R$.

In particular, the number of those of the isometry classes $\mathcal{C}(n, V)$ of CAT(1)-spaces defined in Section 1 is bounded above by a constant depending only on $n$ and $V$.

Acknowledgments. This work was done while the author stayed in the Graduate School of Mathematics, Kyushu University, except for the work in Section 5. The author would like to thank Professor Takao Yamaguchi for his advice and constant encouragement, and thank Professors Yukio Otsu, Katsuhiro Shiohama, and Takashi Shioya for valuable discussions.

The author would also like to thank the referee for his comment and suggestion about the formulation of Theorem 5.7.

\section{References}

[1] A.D. Alexandrov, Uber eine verallgemeinerung der Riemannshen geometrie, Scriften Forschungsinst. Math., 1 (1957), 33-84.

[2] A.D. Alexandrov, V.N. Berestovskii and I.G. Nikolaev, Generalized Riemannian spaces, Uspekhi Mat. Nauk., 41(3) (1986), 3-44; translation in Russian Math. Surveys, 41(3) (1986), 1-54. 
[3] W. Ballmann, Lectures on Spaces of Nonpositive Curvature, DMV-seminar, 25, Birkhäuser, Basel-Boston-Berlin, 1995, MR 97a:53053, Zbl 0834.53003.

[4] W. Ballmann and M. Brin, Orbihedra of nonpositive curvature, Publ. Math. I.H.E.S., 82 (1995), 169-209, MR 97i:53049, Zbl 0866.53029.

[5] _ Diameter rigidity of spherical polyhedra, Duke Math. J., 97(2) (1999), 235259, MR 2000c:53047, Zbl 0980.53045.

[6] S. Barré, Polyèdres finis de dimension 2 à courbure $\leq 0$ et de rang 2, Ann. Inst. Fourier (Grenoble), 45(4) (1995), 1037-1059, MR 96k:53056, Zbl 0831.53031.

[7] M.R. Bridson and A. Haefliger, Metric Spaces of Non-positive Curvature, Grundl. Math. Wissen, 319, Springer-Verlag, Berlin-Heidelberg-New York, 1999, MR 2000k:53038.

[8] Yu.D. Burago and S.V. Buyalo, Metrics of upper bounded curvature on 2-polyhedra II, St. Petersburg Math. J., 10(4) (1999), 619-650, MR 99j:53086, Zbl 0929.52009.

[9] L. Coghlan and Y. Itokawa, A sphere theorem for reverse volume pinching on even-dimensional manifolds, Proc. Amer. Math. Soc., 111(3) (1991), 815-819, MR 91f:53033, Zbl 0719.53019.

[10] M. Gromov, Structures Métriques pour les Variétés Riemanniennes, rédigé par J. Lafontaine et P. Pansu, Cedic/Fernand Nathan, 1981, MR 85e:53051, Zbl 0509.53034.

[11] _ Geometric Group Theory, Essays in group theory (S.M. Gersten, ed.), M.S.R.I. Publ. 8, Springer-Verlag, Berlin-Heidelberg-New York, 1987, 75-264.

[12] B. Kleiner, The local structure of length spaces with curvature bounded above, Math. Z., 231 (1999), 409-456, MR 2000m:53053, Zbl 0940.53024.

[13] B. Kleiner and B. Leeb, Rigidity of quasi-isometries for symmetric spaces and Euclidean buildings, Publ. Math. I.H.E.S., 86 (1997), 115-197, MR 98m:53068, Zbl 0910.53035.

[14] K. Nagano, Asymptotic rigidity of Hadamard 2-spaces, J. Math. Soc. Japan, 52(4) (2000), 699-723, MR 2001f:53081, Zbl 0984.53014.

[15] _ A volume convergence theorem for Alexandrov spaces with curvature bounded above. Preprint (a revised version), 2001, to appear in Math. Z.

[16] Y. Otsu, Differential geometric aspects of Alexandrov spaces, in 'Comparison Geometry' (K. Grove and P. Petersen, eds.), M.S.R.I. Publ. 30, Cambridge Univ. Press, 1997, 135-148, MR 98d:53046, Zbl 0891.53025.

[17] Y. Otsu and H. Tanoue, The Riemannian structure of Alexandrov spaces with curvature bounded above. Preprint.

[18] Yu.G. Perelman, A. D. Aleksandrov's spaces with curvature bounded below II. Preprint, 1991.

[19] Yu.G. Reshetnyak, On the theory of spaces of curvature not greater than $K$ [Russian], Mat. Sb., 52 (1960), 789-798.

[20] G. de Rham, Complexes à automorphismes et homéomorphie différentiable. Ann. Inst. Fourier (Grenoble), 2 (1950), 51-67, MR 13,268c, Zbl 0043.17601.

[21] T.B. Rushing, Topological Embeddings, Pure and Applied Math., 52, Academic Press, New York and London, 1973, MR 50 \#1247, Zbl 0295.57003.

[22] K. Shiohama, Sphere theorems, in 'Handbook of differential geometry, Vol. I' (F.J.E. Dillen and L.C.A. Verstraelen, eds.), North-Holland, Amsterdam, 2000, 865-903, MR 2001c:53051, Zbl 0968.53003. 
[23] J. Tits, Buildings of Spherical Type and BN-Pairs, Lecture Notes in Math., 386, Springer-Verlag, Berlin-Heidelberg-New York, 1974, MR 57 \#9866, Zbl 0295.20047.

Received February 7, 2001 and revised July 20, 2001. This work was partially supported by the Research Fellowships of the Japan Society for the Promotion of Science for Young Scientists.

Mathematical Institute

TOHOKU UNIVERSITY

Aoba, Sendai, Miyagi

980-8578 JAPAN

E-mail address: nagano@math.tohoku.ac.jp 